\title{
Lactancia prolongada y desarrollo del lenguaje: una revisión de la literatura ${ }^{1}$
}

\author{
Gittens Dixon, Katty ${ }^{2}$
}

Institución: Hospital San Rafael de Alajuela

\section{RESUMEN}

Introducción. La lactancia materna es un proceso natural determinante para la supervivencia pero, ¿su prolongación podría perjudicar el desarrollo del lenguaje? El objetivo es determinar la existencia de evidencia científica sobre efectos adversos o perjudiciales de la lactancia materna o natural prolongada en el desarrollo del lenguaje en infantes de dos o más años.

Métodos. Se trata de una investigación secundaria en la que se revisa literatura de las siguientes bases de datos: Annual Reviews, Clinical Key, EBSCO, EBSCOHOST, Google Académico, JAMA Network, Ovid y PubMed, hasta obtener 100 artículos. Una vez analizados y aplicados los criterios de inclusión, se seleccionó 19 documentos para dar respuesta a la pregunta PICO.

Resultados. Según el diseño de los estudios, nueve de ellos presenta un nivel de evidencia II-2, ya que fueron estudios de cohortes y de estudios de casos y controles; luego, siete estuvieron constituidos por revisiones de literatura, de las cuales cuatro fueron revisiones sistemáticas; dos se califican en nivel III de evidencia pues fueron estudios descriptivos, uno mixto descriptivo y otro cualitativo $\mathrm{y}$, por último, uno estuvo constituido por un ensayo clínico que corresponde al nivel de evidencia I.

Conclusiones. La lactancia materna tiene un efecto positivo sobre el desarrollo del lenguaje: existe una relación causal entre duración de la lactancia y el lenguaje receptivo y la inteligencia verbal y no verbal.

Palabras clave: desarrollo-del-lenguaje, lactancia-materna, lactancia-prolongada

\footnotetext{
${ }^{1}$ Fecha de recepción: 22 agosto 2016

Fecha de aceptación: 4 noviembre del 2016

${ }^{2}$ Enfermera obstetra. Hospital San Rafael de Alajuela. Caja Costarricense de Seguro Social. Costa Rica. Correo electrónico:

kattyvanessa@yahoo.com
} 


\title{
Extended breastfeeding and language development: a review of literatura ${ }^{1}$
}

Institution: San Rafael de Alajuela Hospital

\author{
Gittens Dixon, Katty ${ }^{2}$
}

\begin{abstract}
Introduction. Breastfeeding is a determinant for survival but natural process, its extension could harm the development of language? The objective is to determine the existence of scientific evidence on adverse or harmful effects of maternal or prolonged breastfeeding language development in infants of two or more years.

Method. This is a secondary research literature in which the following databases are reviewed: Annual Reviews, Clinical Key, EBSCO, EBSCOHOST, Google Scholar, JAMA Network, Ovid and PubMed, to obtain 100 items. Once analyzed and applied the inclusion criteria, 19 papers were selected to answer the question PICO.

Results. According to the design of the studies, nine of them present a level of evidence II-2, since they were cohort studies and case-control studies; then seven were constituted by literature reviews, of which four were systematic reviews; two are classified in level III of evidence because they were descriptive studies, a mixed descriptive and a qualitative one and, finally, one was constituted by a clinical trial that corresponds to the level of evidence I.
\end{abstract}

Conclusions. Breastfeeding has a positive effect on language development: there is a causal relationship between duration of breastfeeding and receptive language and verbal and nonverbal intelligence.

Keywords: breastfeeding, language-development, prolonged-breastfeeding

\footnotetext{
${ }^{1}$ Date of receipt: August 22, 2016

Date of acceptance: November 4, 2016

${ }^{2}$ Midwife. San Rafael de Alajuela Hospital. Caja Costarricense de Seguro Social. Costa Rica. E-mail: kattyvanessa@yahoo.com
} 


\title{
Lactância prolongada e desenvolvimento da linguagem: uma revisão da literatura ${ }^{1}$
}

\author{
Gittens Dixon, Katty ${ }^{2}$
}

Instituição: Hospital San Rafael de Alajuela

\section{RESUMO}

Introdução. A lactância materna é um processo natural determinante para a sobrevivência, porém, seu prologamento poderia prejudicar o desenvolvimento da linguagem? O objetivo é determinar a existência de evidência científica sobre efeitos adversos ou prejudiciais da lactância materna ou natural prolongada no desenvolvimento da linguagem em crianças de dois ou mais anos.

Métodos. Trata-se de uma pesquisa secundária na que se revisa literatura das seguintes bases de dados: Annual Reviews, Clinical Key, EBSCO, EBSCOHOST, Google Acadêmico, JAMA Network, Ovid e PubMed, até obter 100 artigos. Uma vez analisados e aplicados os critérios de inclusão, se selecionaram 19 documentos para dar resposta a pergunta formulada.

Resultados. De acordo com o desenho do estudo, nove deles apresenta um nível de evidência II-2, assim como os estudos de coorte e estudos de caso-controle; em seguida, sete foram constituídas por revisões de literatura, dos quais quatro eram revisões sistemáticas; tanto qualificar a nível III provas porque eram estudos descritivos, uma mista e uma qualitativa descritiva e, por fim, uma consistiu de um ensaio clínico que corresponde ao nível de evidência I.

Conclusões. A lactância materna tem um efeito positivo sobre o desenvolvimento da linguagem: existe uma relação causal entre duração da lactância e a linguagem receptiva e a inteligência verbal e não verbal.

Palavras-chave: amamentação, amamentação-prolongada, desenvolvimento-da-linguagem

\footnotetext{
${ }^{1}$ Data de recebimento: 22 de agosto de 2016

Data de aceitação: 04 de novembro de 2016

${ }^{2}$ OB enfermeira. Hospital San Rafael de Alajuela. Caja Costarricense de Seguro Social. Costa Rica. Correio eletrônico:

kattyvanessa@yahoo.com
} 


\section{INTRODUCCIÓN}

La lactancia es una de las características principales que identifica a los mamíferos, a cuyo grupo pertenecen los seres humanos, la cual puede significar la diferencia entre la vida y la muerte de las crías. Según datos de la Organización Mundial de la Salud y el Fondo de las Naciones Unidad para la Infancia (UNICEF por las siglas en inglés de United Nations Children's Fund), la lactancia materna óptima en infantes hasta los dos años, potencia más beneficios que cualquier otro tipo de intervención ${ }^{1-2}$.

Por este hecho, dichos organismos internacionales, así como diferentes y diversas asociaciones profesionales recomiendan el inicio temprano de la lactancia (en la primera hora de vida), su exclusividad durante los primeros seis meses (que constituya la principal fuente de aporte calórico para infantes de 6 a 12 meses) y que la leche materna se continúe hasta los dos años o más como complemento nutricional ${ }^{1-2}$.

Si bien, el factor nutricional es de particular relevancia en los lactantes pequeños, mas no es lo único: existe sustento científico sólido y abundante sobre los efectos de la lactancia materna sobre el crecimiento, protección en contra de infecciones y enfermedades, desarrollo motor, el desarrollo psicológico, procesos de aprendizaje, sociales, de construcción de la personalidad y de dentición adecuados y su incidencia en el desarrollo lingüístico. Incluso, hay proyecciones sobre la incidencia de la lactancia materna en el futuro económico de personas adultas que han sido amamantados en comparación con quienes recibieron lactancia artificial $^{1-2-3-4}$.

En el caso específico de la especie humana, desde la introducción de las leches industrializadas, el proceso de lactancia natural ha cedido parte de su espacio al uso de sucedáneos o sustitutos de la leche materna, al punto de que "menos de uno de cada cinco niños es amamantado a los 12 meses en países de alto ingreso y dos de cada tres niños entre los 6 meses y los 2 años recibe alguna cantidad de leche materna en países de bajo y mediano ingreso" 4 .5.

La sustitución de la lactancia materna es un fenómeno multifactorial en la que inciden factores de cada individuo (malformaciones o patologías que dificulten la succión), dificultad para el amamantamiento, la autodeterminación de las mujeres, la ausencia de apoyo familiar, social y legal (por ejemplo, al reincorporarse al trabajo remunerado), la presión de pares y grupos pero, también la indicación, prescripción o presión por parte de profesionales de la salud para introducir sucedáneos.

Considerando que las organizaciones mundiales recomiendan la lactancia materna hasta los dos años o más, se crea la impresión de que después de los dos años podría no ser necesaria por lo que, en la literatura, es llamada lactancia materna prolongada ${ }^{3}$. En algunos casos, según argumentos utilizados por profesionales de salud, en las áreas de medicina, tanto general como especializada, psicología y terapia del lenguaje, entre otras, la lactancia materna prolongada incluso podría ser perjudicial.

El objetivo principal de esta investigación secundaria es determinar la existencia de evidencia científica sobre efectos adversos o perjudiciales de la lactancia materna o natural prolongada relacionados con el desarrollo del lenguaje en infantes de dos o más años. Para esta revisión, se entiende por lactancia materna prolongada la colocación del niño o niña de dos o más años al pecho materno con el propósito de amamantar, indistintamente de 
la frecuencia y duración en tiempo de la tetada ${ }^{6}$. Para concretar tal propósito, la revisión se sustentó en los principios de la Práctica Clínica basada en Evidencia. desde la que se plantea la siguiente pregunta PICO: en infantes con lactancia materna prolongada en comparación con aquellos que fueron destetados antes de los dos años, ¿existe un aumento en el riesgo de desarrollo inadecuado de los procesos del habla?

\section{MATERIALES Y MÉTODOS}

Para esta revisión breve de literatura, basada en la metodología sugerida para la práctica clínica basada en la evidencia, se planteó una pregunta clínica utilizando para ello el acrónimo PICO [P= población, I=intervención, $\mathrm{C}=$ comparación, $\mathrm{O}=$ outcome (resultado esperado)], desglosado de la siguiente manera: en infantes con lactancia materna prolongada en comparación con aquellos que fueron destetados antes de los dos años, ¿existe un aumento en el riesgo de desarrollo inadecuado de los procesos del habla?

La pregunta clínica planteada es de primera línea de tipo causalidad y/o etiología. Los estudios que responden específicamente a la interrogante son de cohorte y estudios de caso y control.

Para la recolección de investigaciones, se realizó búsquedas en las siguientes bases de datos (en orden alfabético y no por orden de importancia): Annual Reviews, Clinical Key, EBSCO, EBSCOHOST, Google Académico, JAMA Network, Ovid y PubMed. Se restringió la búsqueda a los idiomas inglés y español y se delimitó a un periodo de tiempo de 5 años (2011 a 2016). Por otra parte, se utilizó las siguientes palabras claves o descriptores: "extended breastfeeding [AND] language", "extended breastfeeding [AND] speech", "prolonged breastfeeding [AND] language" "prolonged breastfeeding [AND] speech", "long-term breastfeeding [AND] language", "long-term breastfeeding [AND] speech", "sustain breastfeeding [AND] language", "sustain breastfeeding [AND] speech" "breastfeeding [AND] language", "breastfeeding [AND] speech", "language [AND] breastfeeding". Para el idioma español: "lactancia prolongada y lenguaje", "lactancia extendida y lenguaje", "lactancia y lenguaje".

Se recuperó un total de 900 estudios que fueron seleccionados, primeramente, por título quedando 100, los cuales fueron organizados en una matriz de datos del programa Excel. Los datos obtenidos de cada estudio incluyen título, autoría, año, tipo de investigación, objetivo principal, conclusión principal y base de datos donde se encuentra disponible.

Posteriormente, la autora realizó un proceso de depuración de datos a partir del resumen de las investigaciones, cuyo principal criterio de inclusión fue la consideración de lactancia materna de dos o más años, quedando 42 documentos. Los datos resultantes fueron evaluados por medio de la aplicación de los instrumentos de Critical Appraisal Skills Programme en español, obtenidos del dominio electrónico http://www.redcaspe.org. . $^{7}$ 8-9-10-11. Una vez aplicadas las plantillas CASPe al texto completo de los estudios, se descartó un total de 23 investigaciones, debido a que, si bien se estudiaban los beneficios a largo plazo de la lactancia y su incidencia en el desarrollo del lenguaje, las evaluaciones del estado de amamantamiento fueron previas a los 2 años de edad o los datos recolectados en rangos de edad excluían la posibilidad de amamantamiento después de los 24 meses, por tanto, no cumplían el criterio de lactancia prolongada. En cuanto a las 19 publicaciones restantes, se les calificó según niveles de evidencia y grados de recomendación de acuerdo con Canadian Task Force on 


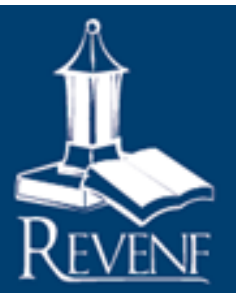

Revista Electrónica Enfermería Actual en costa Rica

Preventive Health Care (CTFPHC) ${ }^{12}$. La figura 1 resume el procedimiento metodológico de dicha revisión literaria.

\section{Figura 1. Diagrama de flujo de revisión de literatura.}
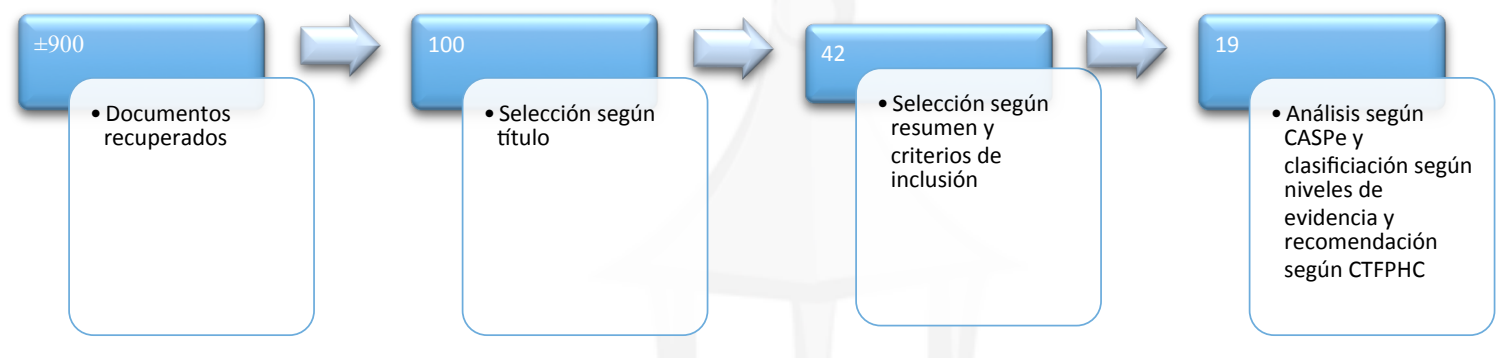

Retomando la metodología de práctica clínica basada en la evidencia, se debe implementar la evidencia y finalmente evaluarla. Para este estudio, la evidencia será divulgada en sesiones clínicas con el personal del Servicio de Obstetricia del Hospital San Rafael de Alajuela, como una actualización en materia de lactancia materna. La evaluación contempla la cantidad de personal actualizado con el resultado de esta revisión breve.

\section{Consideraciones éticas}

La autora respetó los derechos de autor de los artículos revisados y utilizados, evidenciado en la citación de este documento.

\section{RESULTADOS}

De los 19 artículos sometidos a análisis crítico, se descartan 10 de ellos por múltiples aspectos metodológicos y además no respondían a la pregunta clínica planteada. En la tabla 1 se presentan los 9 documentos analizados e incluidos que responden directamente al questionamiento de esta revisión breve. 
Tabla 1. Evaluación de los documentos incluidos.

\begin{tabular}{|c|c|c|c|c|c|}
\hline Título & Año & $\begin{array}{c}\text { Referencia } \\
\text { bibliográfica }\end{array}$ & $\begin{array}{c}\text { Tipo de } \\
\text { investigación }\end{array}$ & $\begin{array}{c}\text { Objetivo y } \\
\text { conclusiones }\end{array}$ & $\begin{array}{l}\text { Resultado del proceso de } \\
\text { lectura crítica según } \\
\text { Canadian Task Force on } \\
\text { Preventive Health Care. }\end{array}$ \\
\hline $\begin{array}{l}\text { Efecto de la } \\
\text { lactancia } \\
\text { materna en } \\
\text { el desarrollo } \\
\text { de niños y } \\
\text { niñas de } 18 \\
\text { a } 4 \text { años. }\end{array}$ & 2014 & $\begin{array}{l}\text { 13. Fernández-Manrique, } \\
\text { MG. Efecto de la lactancia } \\
\text { materna en el desarrollo de } \\
\text { niños y niñas de } 18 \text { a } 4 \text { años. } \\
\text { 2014. Disponible en: } \\
\text { http://repositorio.usfq.edu.e } \\
\text { c/bitstream/23000/3655/1/1 } \\
\text { 11219.pdf }\end{array}$ & $\begin{array}{l}\text { Corte trasversal, de } \\
\text { análisis prospectivo }\end{array}$ & $\begin{array}{l}\text { Objetivo: Investigar la relación entre exposición a la lactancia } \\
\text { materna y los cuatro hitos del desarrollo: motor grueso, motor } \\
\text { fino, lenguaje y habilidad personal social. / Conclusión: } \\
\text { Beneficio de la lactancia materna para el desarrollo motor fino } \\
\text { en los niños medidos a través de la prueba de Denver. En motor } \\
\text { grueso y personal/social, efecto protector sin significancia } \\
\text { estadística. }\end{array}$ & $\begin{array}{l}\text { Nivel de evidencia según } \\
\text { diseño del estudio: II-2. } \\
\text { Nivel de evidencia según } \\
\text { validez interna: bueno. } \\
\text { Grado de recomendación: } \\
\text { I. }\end{array}$ \\
\hline $\begin{array}{l}\text { Relación de } \\
\text { la lactancia } \\
\text { materna y el } \\
\text { desarrollo } \\
\text { Dento-Buco- } \\
\text { Máxilo- } \\
\text { Facial: } \\
\text { Revisión de } \\
\text { la literatura } \\
\text { latinoameric } \\
\text { ana. }\end{array}$ & 2012 & $\begin{array}{l}\text { 24. Rondón, R, Zambrano, } \\
\text { G y Guerra, ME. Relación } \\
\text { de la lactancia materna y el } \\
\text { desarrollo Dento-Buco- } \\
\text { Máxilo-Facial: Revisión de } \\
\text { la literatura latinoamericana. } \\
\text { 2012. Disponible en: } \\
\text { http://ortodoncia.ws/publica } \\
\text { ciones/2012/pdf/art20.pdf }\end{array}$ & $\begin{array}{l}\text { Revisión de } \\
\text { literatura } \\
\text { latinoamericana }\end{array}$ & $\begin{array}{l}\text { Objetivo: Establecer relación entre la lactancia materna y su } \\
\text { impacto en el desarrollo dento-buco-máxilo-facial y su relación } \\
\text { con la presencia de hábitos parafuncionales y la prevención de } \\
\text { maloclusiones. /. Conclusión: Efecto positivo de la lactancia en } \\
\text { la sincronización de las funciones del aparato bucal: succión, } \\
\text { deglución, respiración, masticación y fonación. Niños } \\
\text { amamantados por más de } 9 \text { meses tenían menos probabilidades } \\
\text { de desarrollar problemas fonéticos posteriores. }\end{array}$ & $\begin{array}{l}\text { Nivel de evidencia según } \\
\text { validez interna: bueno. } \\
\text { Grado de recomendacion: } \\
\text { B }\end{array}$ \\
\hline $\begin{array}{l}\text { Lactancia } \\
\text { materna y } \\
\text { resultados } \\
\text { del } \\
\text { desarrollo } \\
\text { del lenguaje: } \\
\text { Una revisión } \\
\text { de la } \\
\text { literatura. }\end{array}$ & 2015 & $\begin{array}{l}\text { 20. Smith, JM. } \\
\text { Breastfeeding and language } \\
\text { outcomes: a review of } \\
\text { literature. Journal of } \\
\text { Communication Disorders } \\
57(2015) \text { 29-40. }\end{array}$ & $\begin{array}{l}\text { Revisión de } \\
\text { literatura. }\end{array}$ & $\begin{array}{l}\text { Objetivo: Realizar una revisión de los estudios existentes que } \\
\text { relacionan la dieta durante la infancia y sus efectos en los } \\
\text { procesos de comunicación. / Conclusión: Existen prejuicios que } \\
\text { pueden sesgar las recomendaciones en cuanto a la lactancia. } \\
\text { Existe evidencia de mecanismos potenciales mediante los } \\
\text { cuales la lactancia incide en el desarrollo neurológico. }\end{array}$ & $\begin{array}{l}\text { Nivel de evidencia según } \\
\text { validez interna: insuficiente. } \\
\text { Grado de recomendación: } \\
\text { C. }\end{array}$ \\
\hline $\begin{array}{l}\text { Riesgo de } \\
\text { déficits en el } \\
\text { desarrollo en } \\
\text { los } \\
\text { prematuros } \\
\text { tardíos: } \\
\text { evaluación a } \\
\text { los } 48 \text { meses } \\
\text { mediante el } \\
\text { Ages \& } \\
\text { Stages } \\
\text { Questionnair } \\
\text { es® }\end{array}$ & 2011 & $\begin{array}{l}\text { 15. Demestre, X, } \\
\text { Schonhaut, L, Morillas, J, } \\
\text { Martínez-Nadal,S, Vila, C, } \\
\text { Raspall, F y Sala, P. Riesgo } \\
\text { de déficit se e el desarrollo } \\
\text { en los prematuros tardíos: } \\
\text { evaluación a los } 48 \text { meses } \\
\text { mediante el Ages \& Stages } \\
\text { Questionnaires. } 2015 . \\
\text { Disponible en: } \\
\text { http://www.analesdepediatri } \\
\text { a.org }\end{array}$ & $\begin{array}{l}\text { Cohorte, } \\
\text { reprospectivo. }\end{array}$ & $\begin{array}{l}\text { Objetivo: Evaluar repercusión de la prematuridad tardía en el } \\
\text { desarrollo psicomotor en niños de } 4 \text { años y compararlo con los } \\
\text { nacidos a término. / Conclusión: Hubo una mayor prevalencia } \\
\text { de riesgo de déficit en el desarrollo en los pretérminos, lo que } \\
\text { justifica considerar esta población de riesgo y establecer } \\
\text { programas de seguimiento eficientes. }\end{array}$ & $\begin{array}{l}\text { Nivel de evidencia según } \\
\text { diseño del estudio: II-2. } \\
\text { Nivel de evidencia según } \\
\text { validez interna: bueno. } \\
\text { Grado de recomendación: } \\
\text { I. }\end{array}$ \\
\hline
\end{tabular}




\begin{tabular}{|c|c|c|c|c|c|}
\hline $\begin{array}{l}\text { La lactancia } \\
\text { materna está } \\
\text { asociada con } \\
\text { una mejoría } \\
\text { en el } \\
\text { desarrollo la } \\
\text { cognición } \\
\text { infantil: Un } \\
\text { estudio de } \\
\text { cohorte de } \\
\text { base } \\
\text { poblacional }\end{array}$ & 2012 & $\begin{array}{l}\text { 29. Quigley, MA, Hockley, } \\
\text { C, Carson, C, Kelly, Y, } \\
\text { Renfrew, MJ y Sacker, A. } \\
\text { Breastfeeding is Associated } \\
\text { with Improved Child } \\
\text { Cognitive Development: A } \\
\text { Population-Based Cohort } \\
\text { Study. 2012. Disponible en: } \\
\text { http://www.sciencedirect.co } \\
\text { m.ezproxy.sibdi.ucr.ac.cr:20 } \\
\text { 48/science/article/pii/S0022 } \\
\text { 347611006627 }\end{array}$ & $\begin{array}{l}\text { Cohorte de base } \\
\text { poblacional. }\end{array}$ & $\begin{array}{l}\text { Objetivo: Evaluar la asociación entre la lactancia materna y el } \\
\text { desarrollo cognitivo en niños de término y pretérmino. / } \\
\text { Conclusión: En hijos únicos, de raza blanca del Reino Unido, la } \\
\text { lactancia materna está asociada con mejorías en el desarrollo } \\
\text { cognitivo, particularmente en niños nacidos de pretérmino. }\end{array}$ & $\begin{array}{l}\text { Nivel de evidencia según } \\
\text { diseño del estudio: II-2. } \\
\text { Nivel de evidencia según } \\
\text { validez interna: bueno. } \\
\text { Grado de recomendación: } \\
\text { I. }\end{array}$ \\
\hline $\begin{array}{l}\text { Duración de } \\
\text { la lactancia } \\
\text { materna y la } \\
\text { habilidad } \\
\text { para el } \\
\text { lenguaje en } \\
\text { la infancia } \\
\text { media. }\end{array}$ & 2011 & $\begin{array}{l}\text { 16. Oddy, W, Li, J, } \\
\text { Robinson, M, Whitehouse, } \\
\text { A. Breastfeeding and early } \\
\text { child development: a } \\
\text { prospective cohort study. } \\
\text { Acta Paediatrica } 2011(100) \\
992-999 .\end{array}$ & Cohorte. & $\begin{array}{l}\text { Objetivo: Determinar si los efectos de la lactancia durante el } \\
\text { desarrollo infantil temprano están presentes en el coeficiente } \\
\text { intelectual, logros académicos y salud mental usando los datos } \\
\text { recolectados de la cohorte Western Australian Pregnancy } \\
\text { Cohort Study. / Conclusión: La mayor duración de la lactancia } \\
\text { materna impresiona tener beneficios significativos en el } \\
\text { coeficiente intelectual, los logros académicos y la salud mental } \\
\text { en niños y adolescentes. }\end{array}$ & $\begin{array}{l}\text { Nivel de evidencia según } \\
\text { diseño del estudio: II-2. } \\
\text { Nivel de evidencia según } \\
\text { validez interna: bueno. } \\
\text { Grado de recomendación: } \\
\text { I. }\end{array}$ \\
\hline $\begin{array}{l}\text { Lactancia } \\
\text { materna por } \\
\text { dos o más } \\
\text { años y su } \\
\text { influencia en } \\
\text { el } \\
\text { crecimiento } \\
\text { y desarrollo } \\
\text { infantil: una } \\
\text { revisión } \\
\text { sistemática }\end{array}$ & 2013 & $\begin{array}{l}\text { 21. Delgado, C y } \\
\text { MatijasevichI, A. } \\
\text { Breastfeeding up to two } \\
\text { years of age or beyond and } \\
\text { its influence on child growth } \\
\text { and development: a } \\
\text { systematic review. } 2013 \text {. } \\
\text { Disponible en: } \\
\text { http://www.scielo.br/scielo. } \\
\text { php?script=sci_isoref\&pid= } \\
\text { S0102- } \\
\text { 311X2013000200012\&lng= } \\
\text { en\&tlng=en }\end{array}$ & $\begin{array}{l}\text { Revisión } \\
\text { sistemática. }\end{array}$ & $\begin{array}{l}\text { Objetivo: Identificar estudios que describan la prevalencia } \\
\text { global de la lactancia hasta los dos años o más y sus efectos en } \\
\text { el crecimiento y desarrollo. / Conclusión: Se concluye que la } \\
\text { evidencia de los efectos a mediano plazo de la lactancia hasta } \\
\text { los dos años o más, son escasos y contradictorios. Por lo tanto, } \\
\text { se requiere de futuras investigaciones con respecto a esta } \\
\text { práctica. }\end{array}$ & $\begin{array}{l}\text { Nivel de evidencia: bueno. } \\
\text { Grado de recomendación: } \\
\text { C. }\end{array}$ \\
\hline $\begin{array}{l}\text { Lactancia } \\
\text { materna, } \\
\text { introducción } \\
\text { de otros } \\
\text { alimentos y } \\
\text { efectos } \\
\text { sobre la } \\
\text { salud: Una } \\
\text { revisión } \\
\text { sistemática } \\
\text { de literatura } \\
\text { para la 5ta. } \\
\text { Edición de } \\
\text { Recomendac } \\
\text { iones } \\
\text { Nutricionale } \\
\text { s Nórdicas. }\end{array}$ & 2013 & $\begin{array}{l}\text { 27. Hörnell, A, Lagström, } \\
\text { H, Lande, B y Thorsdottir, I. } \\
\text { Breastfeeding, introduction } \\
\text { of other foods and effects on } \\
\text { health: a systematic } \\
\text { literature review for the 5th } \\
\text { Nordic Nutrition } \\
\text { Recommendations. } 2013 \text {. } \\
\text { Disponible en: } \\
\text { http://www.ncbi.nlm.nih.go } \\
\text { v/pmc/articles/PMC362570 } \\
\text { 6/ }\end{array}$ & $\begin{array}{l}\text { Revisión } \\
\text { sistemática de } \\
\text { literatura. }\end{array}$ & $\begin{array}{l}\text { Objetivo: Revisar datos científicos válidos en el entorno } \\
\text { nórdico sobre efectos a corto y largo plazo de la lactancia } \\
\text { materna (duración tanto de la lactancia global como la } \\
\text { exclusiva) y la introducción de alimentos complementarios. / } \\
\text { Conclusión: Se encontró evidencia convincente y probable } \\
\text { sobre los beneficios de la lactancia en varios resultados } \\
\text { (outcomes). }\end{array}$ & $\begin{array}{l}\text { Nivel de evidencia según } \\
\text { validez interna: buena. } \\
\text { Nivel de recomendación: } \\
\text { A. }\end{array}$ \\
\hline $\begin{array}{l}\text { Lactancia } \\
\text { materna y } \\
\text { desarrollo } \\
\text { cognitivo - } \\
\text { el rol de } \\
\text { confusores: } \\
\text { Una revisión } \\
\text { sistemática }\end{array}$ & 2013 & $\begin{array}{l}\text { 22. Walfisch, A, Sermer, C, } \\
\text { Cressman, A y Koren, G. } \\
\text { Breast milk and cognitive } \\
\text { development_- the role of } \\
\text { confounders: a systematic } \\
\text { review. 2013. Disponible } \\
\text { en: } \\
\text { http://bmjopen.bmj.com/con } \\
\text { tent/3/8/e003259.full.pdf }\end{array}$ & $\begin{array}{l}\text { Revisión } \\
\text { sistemática de } \\
\text { literatura. }\end{array}$ & $\begin{array}{l}\text { Objetivo: La asociación entre lactancia materna y desarrollo } \\
\text { ccognitivo infantil está en conflicto entre efectos positivos y } \\
\text { nulos. Esta relación puede estar "confundida" por factores } \\
\text { asociados a la lactancia materna, específicamente clase } \\
\text { socioeconómica y coeficiente intelectual maternos. / } \\
\text { Conclusión: Muchos de los efectos de la lactancia materna en el } \\
\text { desarrollo neurológico infantil reportados, se deben a factores } \\
\text { confusores. Es poco probable que trabajos adicionales cambien } \\
\text { esta síntesis. }\end{array}$ & $\begin{array}{l}\text { Nivel de evidencia según } \\
\text { validez interna: insuficiente. } \\
\text { Grado de recomendación: } \\
\text { C. }\end{array}$ \\
\hline
\end{tabular}




\section{Revista Electrónica Enfermeria Actual en costa Rica}

\begin{tabular}{|c|c|c|c|c|c|}
\hline $\begin{array}{l}\text { Alimentació } \\
\mathrm{n} \text { infantil y } \\
\text { desarrollo } \\
\text { cognitivo } \\
\text { infantil a los } \\
3 \text { y } 7 \text { años } \\
\text { de edad: } \\
\text { efectos de la } \\
\text { duración de } \\
\text { lactancia } \\
\text { materna y su } \\
\text { exclusividad } \\
\text {. }\end{array}$ & 2013 & $\begin{array}{l}\text { 17. Belfort MB, Rifas- } \\
\text { Shiman SL, Kleinman KP, } \\
\text { Guthrie LB, Bellinger DC, } \\
\text { Taveras EM, et al. Infant } \\
\text { Feeding and Childhood } \\
\text { Cognition at Ages } 3 \text { and } 7 \\
\text { Years. } \\
\text { Effects of Breastfeeding } \\
\text { Duration and Exclusivity. } \\
\text { 2013. Disponible en: } \\
\text { http://archpedi.jamanetwork } \\
\text {.com/article.aspx?utm_camp } \\
\text { aign=2013-08- } \\
\text { 09\%20hvnews.htm1\&utm_- } \\
\text { medium=email\&utm_source } \\
=\text { eloqua\&articleid=1720224 } \\
\text { \&elq=625d84fecb7f478db2 } \\
\text { 114fd61d3e27e3\&elqcampa } \\
\text { ignid=138 }\end{array}$ & $\begin{array}{l}\text { Cohorte, } \\
\text { prospectivo. }\end{array}$ & $\begin{array}{l}\text { Objetivo: Examinar las relaciones entre la duración y } \\
\text { exclusividad de la lactancia materna con la cognición infantil a } \\
\text { los } 3 \text { y } 7 \text { años. / Conclusión: Los resultados sustentan una } \\
\text { relación causal entre la duración de la lactancia materna con el } \\
\text { lenguaje receptivo y la inteligencia verbal y no verbal más } \\
\text { adelante en la vida. }\end{array}$ & $\begin{array}{l}\text { Nivel de evidencia según } \\
\text { diseño del estudio: II-2. } \\
\text { Nivel de evidencia según } \\
\text { validez interna: buena. } \\
\text { Grado de recomendación: } \\
\text { I. }\end{array}$ \\
\hline $\begin{array}{l}\text { ¿Contribuirá } \\
\text { la lactancia } \\
\text { materna a la } \\
\text { brecha racial } \\
\text { en } \\
\text { evaluaciones } \\
\text { de lectura y } \\
\text { matemáticas } \\
?\end{array}$ & 2013 & $\begin{array}{l}\text { 18. Peters, K.E., Huang, J., } \\
\text { Vaughn, M.G. y Witko, C. } \\
\text { Does breastfeeding } \\
\text { contribute to the racial gap } \\
\text { in reading and math test } \\
\text { scores? 2013. Disponible } \\
\text { en: } \\
\text { http://www.ncbi.nlm.nih.go } \\
\text { v/pmc/articles/PMC383620 } \\
\text { 2/ }\end{array}$ & Cohorte. & $\begin{array}{l}\text { Objetivo: Examinar el impacto de prácticas divergentes de } \\
\text { lactancia materna entre madres caucásicas y afroamericanas y } \\
\text { la brecha persistente en las evaluaciones de niños caucásicos y } \\
\text { afroamericanos. / Conclusión: Los hallazgos del estudio indican } \\
\text { que la lactancia materna explican las brechas del } 17 \% \text { y } 9 \% \\
\text { observadas en las puntuaciones de lectura y matemática } \\
\text { respectivamente, entre afroamericanos y caucásicos; un efecto } \\
\text { mayor que la mayoría de las intervenciones recientes en } \\
\text { políticas educativas. Se deben implementar renovados esfuerzos } \\
\text { alrededor de políticas y prácticas clínicas que promuevan y } \\
\text { remuevan barreras a la lactancia materna para las madres } \\
\text { afroamericanas. }\end{array}$ & $\begin{array}{l}\text { Nivel de evidencia según } \\
\text { diseño del estudio: II-2. } \\
\text { Nivel de evidencia según } \\
\text { validez interna: buena. } \\
\text { Grado de recomendación: } \\
\text { I. }\end{array}$ \\
\hline $\begin{array}{l}\text { Definiciones } \\
\text { de lactancia } \\
\text { materna: un } \\
\text { llamado para } \\
\text { el desarrollo } \\
\text { y uso } \\
\text { consistente } \\
\text { de } \\
\text { definiciones } \\
\text { en la } \\
\text { investigació } \\
\text { n y en la } \\
\text { revisión } \\
\text { literaria de } \\
\text { pares. }\end{array}$ & 2012 & $\begin{array}{l}\text { 7. Labbok, M.H. y Starling, } \\
\text { A. Definitions of } \\
\text { Breastfeeding: Call for the } \\
\text { Development and Use of } \\
\text { Consistent Definitions in } \\
\text { Research and Peer- } \\
\text { Reviewed Literature. } 2012 . \\
\text { Disponible en: } \\
\text { http://online.liebertpub.com/ } \\
\text { doi/abs/10.1089/bfm.2012.9 } \\
\text { 975\#/doi/abs/10.1089/bfm.2 } \\
012.9975\end{array}$ & $\begin{array}{l}\text { Revisión } \\
\text { sistemática de } \\
\text { literatura. }\end{array}$ & $\begin{array}{l}\text { Objetivo: Valorar cuales definiciones se lactancia materna han } \\
\text { sido utilizadas en "journals" relevantes y la fuente de dichas } \\
\text { definiciones. / Conclusión: Existe una necesidad de que los } \\
\text { "journals" aumenten los requisitos de inclusión para } \\
\text { definiciones de lactancia y de los investigadores, su uso. Para } \\
\text { que esto suceda, primeramente, debe existir una reconfirmación } \\
\text { y/o desarrollo de una serie de definiciones consistentemente } \\
\text { usada que sea aplicable para estudios de comportamientos, } \\
\text { sustento a intervenciones y de resultados en salud tanto para la } \\
\text { madre como el niño. }\end{array}$ & $\begin{array}{l}\text { Nivel de evidencia según } \\
\text { validez interna: Buena. } \\
\text { Grado de recomendación: } \\
\text { A. }\end{array}$ \\
\hline $\begin{array}{l}\text { Duración de } \\
\text { la lactancia } \\
\text { materna y } \\
\text { habilidad } \\
\text { lingüística } \\
\text { en la } \\
\text { infancia } \\
\text { media. }\end{array}$ & 2013 & $\begin{array}{l}\text { 28. Whitehouse, A.J., } \\
\text { Robinson, M., Li, J. y Oddy, } \\
\text { W.H. Duration of breast } \\
\text { feeding and language ability } \\
\text { in middle childhood. 2013.. } \\
\text { Disponible en: } \\
\text { http://www.ncbi.nlm.nih.go } \\
\text { v/pubmed/21133968 }\end{array}$ & $\begin{array}{l}\text { Cohorte, } \\
\text { longitudinal. }\end{array}$ & $\begin{array}{l}\text { Objetivo: Examinar si las asociaciones positivas de la lactancia } \\
\text { materna sobre las habilidades lingüísticas a la edad de } 5 \text { años de } \\
\text { la cohorte Western Australian Pregnancy (Raine), aun estaban } \\
\text { presentes a los } 10 \text { años de edad. Conclusión: La lactancia por } \\
\text { periodos más largos al inicio de la vida, tiene un efecto positivo } \\
\text { y estadísticamente independiente sobre el desarrollo del } \\
\text { lenguaje en la niñez media. }\end{array}$ & $\begin{array}{l}\text { Nivel de evidencia según } \\
\text { diseño del estudio: II-2. } \\
\text { Nivel de evidencia según } \\
\text { validez interna: buena. } \\
\text { Grado de recomendación: } \\
\text { A. }\end{array}$ \\
\hline
\end{tabular}




\section{Revista Electrónica Enfermeria Actual en costa Rica}

\begin{tabular}{|c|c|c|c|c|c|}
\hline $\begin{array}{l}\text { Especificida } \\
\text { d sexual en } \\
\text { la asociación } \\
\text { entre } \\
\text { lactancia } \\
\text { materna y la } \\
\text { integración } \\
\text { de materia } \\
\text { blanca en } \\
\text { niños de } 8 \\
\text { años de } \\
\text { edad. }\end{array}$ & 2014 & $\begin{array}{l}\text { 19. Ou X, Andres A, Cleves } \\
\text { MA, Pivik RT, Snow JH, } \\
\text { Ding Z et al. Sex-specific } \\
\text { association between infant } \\
\text { diet and white matter } \\
\text { integrity in 8-y-old children. } \\
\text { 2014. Disponible en: } \\
\text { http://www.nature.com/pr/jo } \\
\text { urnal/v76/n6/full/pr2014129 } \\
\text { a.html }\end{array}$ & Ensayo clínico. & $\begin{array}{l}\text { Conclusión: La duración de la lactancia materna fue asociada } \\
\text { con mejor desarrollo de la materia blanca a los } 8 \text { años de edad } \\
\text { en varones. Una asociación similar NO fue observada en las } \\
\text { niñas. }\end{array}$ & $\begin{array}{l}\text { Nivel de evidencia según } \\
\text { diseño del estudio: I. Nivel } \\
\text { de evidencia según validez } \\
\text { interna: buena. Nivel de } \\
\text { recomendación: } \text { I. }\end{array}$ \\
\hline $\begin{array}{l}\text { Periodos } \\
\text { críticos en la } \\
\text { percepción } \\
\text { del habla: } \\
\text { Nuevas } \\
\text { direcciones }\end{array}$ & 2015 & $\begin{array}{l}\text { 30. Werker, JF y Hensk, } \\
\text { TK. Critical periods in } \\
\text { speech perception: New } \\
\text { directions. } 2015 \text {. } \\
\text { Disponible en: } \\
\text { www.annualreviews.org }\end{array}$ & $\begin{array}{l}\text { Revisión de } \\
\text { literatura. }\end{array}$ & $\begin{array}{l}\text { Objetivo: Revisar la literatura sobre el desarrollo de la } \\
\text { percepción del lenguaje humano, en el contexto del modelo de } \\
\text { periodos críticos; resaltando investigaciones que revelen la } \\
\text { interacción de influencias madurativas y vivenciales en } \\
\text { cojunturas claves del desarrollo; y presentar ejemplos } \\
\text { paradigmáticos que prueban el modelo de periodos críticos en } \\
\text { sujetos humanos. Conclusión: Se abre la discusión para } \\
\text { entender cómo una comprensión mecanicista de los periodos } \\
\text { críticos produce cambios en la naturaleza del debate siendo que } \\
\text { la pregunta ya no es si existen los periodos crítiocs sino cuáles } \\
\text { procesos los abren, los mantienen abiertos, los cierran y qué } \\
\text { permite que su reapertura. }\end{array}$ & $\begin{array}{l}\text { Nivel de evidencia según } \\
\text { validez interna: insuficiente. } \\
\text { Grado de recomendación: } \\
\text { I. }\end{array}$ \\
\hline $\begin{array}{l}\text { Barreras de } \\
\text { lactancia } \\
\text { materna y } \\
\text { asociación } \\
\text { de sus } \\
\text { prácticas } \\
\text { con el } \\
\text { desarrollo } \\
\text { psicomotor } \\
\text { en niños de } \\
6 \text { a } 24 \\
\text { meses, Cerro } \\
\text { Papa - Villa } \\
\text { el Salvador. }\end{array}$ & 2015 & $\begin{array}{l}\text { 14. Tintaya-Peña, V. } \\
\text { Barreras de lactancia } \\
\text { materna y asociación de sus } \\
\text { prácticas con el desarrollo } \\
\text { psicomotor en niños de } 6 \text { a } \\
24 \text { meses, Cerro Papa - } \\
\text { Villa el Salvador. } 2015 \text {. } \\
\text { Disponible en: } \\
\text { http://cybertesis.unmsm.edu } \\
\text {.pe/handle/cybertesis/4393 }\end{array}$ & $\begin{array}{l}\text { Investigación de } \\
\text { enfoque Mixto. El } \\
\text { enfoque } \\
\text { cuantitativo tuvo } \\
\text { un diseño } \\
\text { descriptivo de } \\
\text { asociación cruzada, } \\
\text { transversal y } \\
\text { observacional. El } \\
\text { enfoque cualitativo } \\
\text { un diseño de teoría } \\
\text { fundamentada. }\end{array}$ & $\begin{array}{l}\text { Objetivo: Determinar la asociación entre las prácticas de } \\
\text { lactancia materna y el desarrollo psicomotor en niños de } 6 \text { a } 24 \\
\text { meses y explorar las barreras de lactancia materna en madres } \\
\text { con niños de } 6 \text { a } 24 \text { meses. / Conclusión: Se encontró } \\
\text { asociación entre la frecuencia de lactancia materna y el } \\
\text { desarrollo psicomotor; sin embargo para la lactancia materna en } \\
\text { la primera hora de vida, la lactancia materna exclusiva y la } \\
\text { técnica de lactancia materna no se encontró asociación con el } \\
\text { desarrollo psicomotor. }\end{array}$ & $\begin{array}{l}\text { Nivel evidencia según } \\
\text { diseño del estudio: III. } \\
\text { Nivel de evidencia según } \\
\text { validez interna: insuficiente. } \\
\text { Grado de recomendación: } \\
\text { I. }\end{array}$ \\
\hline
\end{tabular}




\begin{tabular}{|c|c|c|c|c|c|}
\hline $\begin{array}{l}\text { Lactancia } \\
\text { materna y su } \\
\text { asociación } \\
\text { con el } \\
\text { rendimiento } \\
\text { académico } \\
\text { en } \\
\text { estudiantes } \\
\text { de } 6^{\circ} \text { y } 7^{\circ} \\
\text { años de } \\
\text { educación } \\
\text { básica en la } \\
\text { Unidad } \\
\text { Educativa } \\
\text { San José la } \\
\text { Salle de la } \\
\text { ciudad de } \\
\text { Latacunga, } \\
\text { Cotopaxi - } \\
\text { Ecuador en } \\
\text { el primer } \\
\text { quimestre } \\
\text { del periodo } \\
\text { escolar } 2015 \\
\text {-2016 }\end{array}$ & 2016 & $\begin{array}{l}\text { 23. Espinosa-Cevallos, YB } \\
\text { y Maldonado-Pacheco, JE. } \\
\text { Lactancia materna y su } \\
\text { asociación con el } \\
\text { rendimiento académico en } \\
\text { estudiantes de } 6^{\circ} \text { y } 7^{\circ} \text { años } \\
\text { de educación básica en la } \\
\text { Unidad Educativa San José } \\
\text { la Salle de la ciudad de } \\
\text { Latacunga, Cotopaxi - } \\
\text { Ecuador en el primer } \\
\text { quimestre del periodo } \\
\text { escolar } 2015 \text {-2016. 2016. } \\
\text { Disponible en: } \\
\text { http://repositorio.puce.edu.e } \\
\text { c/handle/22000/10474 }\end{array}$ & $\begin{array}{l}\text { Estudio de cohorte } \\
\text { histórico. }\end{array}$ & $\begin{array}{l}\text { Objetivo: Establecer si la lactancia materna se asocia con un } \\
\text { buen rendimiento académico en los estudiantes de } 6^{\circ} \text { y } 7^{\circ} \text { años } \\
\text { de educación básica de la Unidad Educativa San José La Salle } \\
\text { de la ciudad de Latacunga en el primer quimestre del periodo } \\
\text { escolar } 2015 \text {-2016. / Conclusión: La asociación entre lactancia } \\
\text { materna y rendimiento escolar no fue estadísticamente } \\
\text { significativa. }(p>0,05) \text {. }\end{array}$ & $\begin{array}{l}\text { Nivel de evidencia según } \\
\text { diseño del estudio: II-2. } \\
\text { Nivel de evidencia según } \\
\text { validez interna: buena. } \\
\text { Grado de recomendación: } \\
\text { C. }\end{array}$ \\
\hline $\begin{array}{l}\text { Riesgo de } \\
\text { sustitución } \\
\text { de la } \\
\text { lactancia } \\
\text { natural por } \\
\text { leche } \\
\text { artificial y } \\
\text { su relación } \\
\text { con el estado } \\
\text { de salud del } \\
\text { lactante que } \\
\text { acude al } \\
\text { servicio de } \\
\text { pediatría } \\
\text { periodo } \\
\text { Abril } 2012 \text { a } \\
\text { Septiembre } \\
\text { del } 2012\end{array}$ & 2012 & $\begin{array}{l}\text { 26. Montoya, V, Córdova, } \\
\text { M y del Cisne, L. Riesgo } \\
\text { de sustitución de la lactancia } \\
\text { natural por leche artificial y } \\
\text { su relación con el estado de } \\
\text { salud del lactante que acude } \\
\text { al servicio de pediatría } \\
\text { periodo Abril } 2012 \text { a } \\
\text { Septiembre del } 2012.2012 \text {. } \\
\text { Disponible en: } \\
\text { http://dspace.unl.edu.ec/jspu } \\
\text { i/handle/123456789/6673 }\end{array}$ & $\begin{array}{l}\text { Observacional, de } \\
\text { corte trasversal. }\end{array}$ & $\begin{array}{l}\text { Objetivo: Determinar si la sustitución de la leche natural por la } \\
\text { leche artificial afecta al lactante en su estado de salud, así como } \\
\text { también en su estado psico-motriz, de igual manera establecer } \\
\text { el grado de conocimiento que tienen las madres sobre la } \\
\text { lactancia materna. / Conclusión: Se determinó que de los niños } \\
(35 \%) \text { alimentados con leche de fórmula; han presentado algún } \\
\text { grado de retraso del desarrollo psico-motriz. }\end{array}$ & $\begin{array}{l}\text { Nivel de evidencia según } \\
\text { graduación del diseño de } \\
\text { estudio: III. Nivel de } \\
\text { evidencia según validez } \\
\text { interna: insuficiente. Grado } \\
\text { de recomendación: B. }\end{array}$ \\
\hline $\begin{array}{l}\text { Influencia } \\
\text { de la } \\
\text { lactancia } \\
\text { materna en } \\
\text { la } \\
\text { prevención } \\
\text { de } \\
\text { maloclusion } \\
\text { es, en } \\
\text { infantes de } 0 \\
\text { a } 36 \text { meses } \\
\text { de edad. }\end{array}$ & 2013 & $\begin{array}{l}\text { 25. Cigüeñas-Raya, EM. } \\
\text { Influencia de la lactancia } \\
\text { materna en la prevención de } \\
\text { maloclusiones, en infantes } \\
\text { de } 0 \text { a } 36 \text { meses de edad. } \\
\text { 2013. Disponible en: } \\
\text { http://200.62.146.130/bitstre } \\
\text { am/cybertesis/3080/3/Cigue } \\
\% \text { C3\%B1as_re.pdf }\end{array}$ & Cohorte. & $\begin{array}{l}\text { Objetivo: Determinar la asociación entre el tipo de lactancia y } \\
\text { el desarrollo de maloclusiones. / Conclusión: Se encontró } \\
\text { diferencias estadísticamente significativas entre los tipos de } \\
\text { lactancia y el desarrollo de maloclusiones, además de un riesgo } \\
\text { relativo de } 1.32 \text { veces más en el tipo de lactancia de biberón } \\
\text { que en el de lactancia materna exclusiva para desarrollar } \\
\text { maloclusiones. }\end{array}$ & $\begin{array}{l}\text { Nivel de evidencia según } \\
\text { graduación del diseño del } \\
\text { estudio: II-2. Nivel de } \\
\text { evidencia según validez } \\
\text { interna: moderada. Grado } \\
\text { de recomendación: B. }\end{array}$ \\
\hline
\end{tabular}

Según el diseño de los estudios, nueve de ellos presenta un nivel de evidencia II-2, ya que fueron estudios de cohortes y de estudios de casos y controles; luego, siete estuvieron constituidos por revisiones de literatura, de las cuales cuatro fueron revisiones sistemáticas; dos se califican en nivel III de evidencia pues fueron estudios 
descriptivos, uno mixto descriptivo y otro cualitativo y, por último, uno estuvo constituido por un ensayo clínico que corresponde al nivel de evidencia I.

Una gran mayoría de los estudios (13) cumplieron los criterios específicos de un estudio bien diseñado por lo que su validez interna se calificó como buena; 5 fueron calificados como insuficiente ya que en el apartado de "Materiales y métodos" no presentaba claridad de que cumplan con los criterios específicos de estudio bien diseñado y, finalmente, un estudio fue clasificado con una validez interna moderada, debido a la utilización de muestreo no probabilístico.

Tomando en cuenta los dos criterios anteriores (graduación según diseño y calidad según la validez interna), se estableció los grados de recomendación para las intervenciones específicas en prevención, de las cuales

- Nueve cuentan con evidencia insuficiente para hacer recomendaciones, sin embargo, otros factores podrían influir en la decisión. En estos estudios, la existencia de lactancia prolongada es inferida ya que se refieren a lactancia después de los 9,12 o 18 meses sin establecer un límite superior de edad a la constante aunque, igualmente, hay una asociación positiva entre la duración de la lactancia y los resultados desarrollo motor fino ${ }^{13-14}$, cognitivo en niños nacidos de pretérmino ${ }^{15}$, rendimiento escolar, coeficiente intelectual y salud mental ${ }^{16}$, lenguaje receptivo e inteligencia verbal y no verbal ${ }^{17}$, puntuación en evaluaciones de lectura y matemática ${ }^{18}$, desarrollo de la materia blanca a nivel cerebral en varones ${ }^{19}$ (grado I, según CTFPHC).

- Cuatro de las investigaciones estiman grado C en CTFPHC, debido a que la evidencia disponible es conflictiva y no permite hacer recomendaciones a favor o en contra de la lactancia en general y menos aún en cuanto a la lactancia prolongada ${ }^{20-21-22-23}$.

- Tres estudios tienen moderada evidencia para recomendar la lactancia materna prolongada (grado B), por incidir en forma indirecta en el lenguaje ${ }^{24-25}$, por tratarse de estudios descriptivos que no determinan causalidad ${ }^{26}$.

- Tres estudios, se clasifican con grado A, puesto que existe buena evidencia para recomendar la lactancia prolongada $^{27-28}$ y porque señala la necesidad de unificar conceptos y definiciones sobre lactancia para futuras investigaciones?

\section{Lactancia materna prolongada y el habla}

La lactancia materna puede ser dificultosa al inicio de la relación entre la boca del recién nacido y la mama. Una vez superadas las dificultades, empieza un proceso complejo de coordinación muscular en la boca del infante para extraer la leche, el cual puede considerarse la base para el posterior desarrollo del habla.

Durante el proceso de succión y deglución, la lengua se contrae repetidamente, lo que conlleva al desarrollo del posicionamiento adecuado para la articulación del sonido de las palabras. De la misma manera, también se ejercitan los músculos orbiculares de los labios, encargados de inmovilizar el pecho antes de comprimirlo y extraer la leche, los cuales son músculos que participan en el cierre labial que permite más adelante 
el soplar y silbar. La mandíbula también juega un papel primordial al permitir la compresión de la mama y la extracción láctea.

De igual forma, la lactancia materna también facilita la respiración nasal en el lactante, cuya práctica incide de manera directa en la capacidad de pronunciar adecuadamente las palabras. Posteriormente, al introducirse los primeros alimentos, la masticación que emplea el mismo grupo muscular propiciará, a partir de movimientos gruesos, el desarrollo de los movimientos finos indispensable para la emisión de sonidos y palabras ${ }^{5}$.

En consecuencia con lo expuesto, Rondón et al. concluyen que existe un efecto positivo de la lactancia en la sincronización de las funciones del aparato bucal, además de un desarrollo dento-buco-maxilo-facial adecuado, por lo tanto, los infantes que son amamantados por más de nueve meses tienen menos probabilidades de desarrollar dificultades posteriores, relacionadas con los sonidos del habla ${ }^{24}$. De igual manera, Cigüeñas-Raya encontró un menor riesgo relativo de desarrollar problemas de mal oclusión en niños amamantados ${ }^{25}$.

En cuanto a la asociación directa entre la lactancia prolongada y su efecto protector en el desarrollo del habla, Fernández-Manrique ${ }^{13}$ lo evidencia a partir del desarrollo motor fino, mientras que Tintaya-Peña ${ }^{14}$, y Montoya et $\mathrm{al}^{26}$ lo asocian con el desarrollo psicomotor y Quigley et $\mathrm{al}^{29}$, con mejoras en el desarrollo cognitivo.

Algunos estudios se orientaron a la presencia o persistencia de dichos beneficios en el largo plazo, tal como en el caso de Oddy et $\mathrm{al}^{16}$, quienes asocian una mayor duración de la lactancia materna con beneficios en el coeficiente intelectual y Whitehouse et $\mathrm{al}^{28}$ observaron que el efecto positivo de la lactancia en el lenguaje persistía en el tiempo, al igual que Belfort et $\mathrm{al}^{17}$.

Por otra parte, se encontró investigaciones que cuestionan los hallazgos positivos en cuanto a la lactancia materna, ya que los asocian a factores confusos, tales como las de Walfisch et al ${ }^{22}$, Delgado et $\mathrm{al}^{21} \mathrm{y} \mathrm{Smith}^{20}$ aunque este último afirma que sí hay evidencia de mecanismos potenciales para la incidencia de la lactancia materna en el desarrollo neurológico.

Por último, Werker ${ }^{30}$ establece que el desarrollo del lenguaje se da durante periodos críticos determinados y que existen procesos que abren y cierran esos periodos. La lactancia podría corresponder a uno de esos periodos y su prolongación podría mantenerlo abierto facilitando el desarrollo lingüístico.

\section{DISCUSIÓN}

Para empezar, es indispensable destacar que, aunque haya indicadores a nivel mundial de bajas tasas de amamantamiento (inicial, exclusivo, duración, etcétera) ${ }^{1-2-4-31}$, la lactancia es un proceso natural en los seres humanos. A pesar de ello, según datos de la UNICEF "solo el 38\% de los niños de menos de seis meses de edad en el mundo en desarrollo recibe leche materna exclusivamente y solo el 39\% de los niños de 20 a 23 meses edad se benefician de la práctica de la lactancia materna",

La sustitución de la lactancia materna por la leche de otras madres (en el mejor de los casos) o la de otros animales no es reciente, sino que data de siglos atrás, como se evidencia en citas bíblicas en las que se habla del papel de las nodrizas o en escritos ancestrales de otras culturas que describen el uso de leche de vacas, cabras, 
ovejas y otros cuando se presentaba algún problema asociado a la lactancia (muerte o enfermedad de la madre o incluso la incapacidad de esta para amamantar $)^{32}$.

Los factores que inciden en el abandono de la lactancia materna se han identificado y estudiados ampliamente y pueden ser agrupados en diversas categorías, mas sin limitarse a estas, tales como las siguientes: condiciones sociales de la madre (inestabilidad en la relación de pareja, ingreso socioeconómico bajo, bajo nivel educativo, falta de apoyo del grupo familiar, reincorporación al ámbito laboral, tenencia de otros hijos e hijas pequeñas o dependientes, etc.); tipo de parto (partos distócicos y cesáreas); problemas con el amamantamiento (mala técnica, pezones adoloridos o agrietados, poca producción láctea o "bajada" tardía de la leche, entre otros); condiciones asociadas al infante (malformaciones que dificulten el acople con el pecho, llanto excesivo, irritabilidad, incluso el sexo, ya que los varones tienden a ser destetados antes que las niñas); falta de redes de apoyo tanto a nivel comunitario como en los servicios de salud ${ }^{14-23-26}$. Además, no se puede olvidar la autodeterminación de la mujer que decide no dar de mamar por diversas razones.

Igualmente, la duración de la lactancia materna o la edad del destete ha sido motivo de controversia y, por lo tanto, también objeto de estudio. En las últimas décadas, ha surgido más apoyo desde la antropología a la duración de la lactancia materna en rangos que van desde los dos años y medio hasta los 7 años según se enfoque desde la ganancia de peso, aumento de la estatura, aparición de la dentición permanente y otros ${ }^{4-31-32-33}$. En este punto surge una encrucijada: algunos autores consideran que la lactancia prolongada va más allá del año, mientras que otros añaden que se extiende por más de 6 meses, es decir, después de completar el periodo de exclusividad recomendado por organismos internacionales, además de que otros lo extienden hasta después de los dos años, por ello. Labbok y Starling ${ }^{7}$ insisten en la necesidad de establecer consenso sobre los conceptos referentes a la lactancia y las definiciones e implicaciones de estas, ya que hay una grama de publicaciones que no pueden ser comparadas fehacientemente por discrepar en elementos básicos de definición e indicadores. Este hecho fue evidente desde el inicio de esta investigación, como se mencionó en el apartado de métodos y materiales, por lo que debieron modificarse las palabras claves para realizar la pesquisa de datos.

Lo anterior, lleva necesariamente al planteamiento de ¿qué debería considerarse como lactancia materna prolongada o extendida? Como designa el Comité de Lactancia Materna de la AEP prologar es hacer que algo dure más de lo normal o establecido, haciendo que la lactancia prolongada "vaya más allá de lo recomendado", lo que ya ha sido refutado, dados los múltiples beneficios tanto para el infante, como para la madre.

Además, otro inconveniente con respecto a las publicaciones realizadas en torno a temas de lactancia, su duración, exclusividad y efectos sobre la salud materno-infantil es la falta de control de confusores que pueden estar llevando a atribuir a la lactancia materna efectos que están asociados a ella como lo son el estímulo materno, el ambiente protector en que se da el amamantamiento, entre otros ${ }^{22-34}$.

A pesar de estas inconsistencias en los procesos de investigación con respecto a la lactancia, existe evidencia de peso en cuanto a sus efectos beneficiosos al punto de que la OMS declara "una lactancia materna óptima tiene tal importancia que permitiría salvar la vida de más de 800000 menores de 5 años todos los años"2. Siendo así, ¿qué impulsa a los profesionales de la salud a recomendar el destete? ${ }^{14-26-31}$. A dicha interrogante, Cockerham-Colas et $\mathrm{al}^{31}$, parecen responder con su estudio al concluir que, en primer lugar, existe una deficiencia en la formación de profesionales en salud en torno a la lactancia pero, sobre todo, existe una actitud muy negativa 
de parte del personal de salud por la lactancia "prolongada" (ya que se aclara que esta clasificación debería desaparecer): a mayor duración, la actitud es más negativa, ya que pasa de un $35 \%$-si el infante tienen de 1 a 2 años- a $65 \%$-si tienen de 3 a 4 años.

Los profesionales de la salud establecen un prejuicio de daño psicológico por la duración de la lactancia, además, presentan una sensación de vergüenza o incomodidad en caso de que una mujer amamante a su hijo o hija (de un $30 \%$ en niños de 1 a 2 años, y de un 55\% en niños de 3 a 4 años). También encontraron que aquellos profesionales que no eran padres o madres tenían actitudes más desfavorables por la lactancia "prolongada" que aquellos que tenían hijos (52\% y $28 \%$ respectivamente). Respecto de lo mencionado, los profesionales en el campo de la salud somos los llamados a responder a las dudas y consultas de las usuarias y a brindar apoyo y asesoría en cuanto a los temas concernientes a la salud, específicamente en este caso, sobre lactancia, motivo por el que es indispensable explorar los conocimientos y capacidades, además de las actitudes y prejuicios que inciden en una práctica clínica, no solamente sustentada en la tradición, sino en la ciencia misma.

\section{CONCLUSIONES}

La lactancia materna sigue siendo una fuente de alimentación, vinculación y estimulación física, emocional y del desarrollo para infantes, indistintamente de su edad. Aunque existe un vacío investigativo en el área específica entre la incidencia de la lactancia materna más allá de los dos o más años y el desarrollo del lenguaje, no hay evidencia que sustente la indicación o recomendación de suspender la lactancia para evitar el desarrollo inadecuado del habla. Existe la necesidad de unificar criterios en cuanto a definiciones y metodologías de investigación en temas de lactancia.

\section{Conflicto de intereses}

El equipo de investigación declara no tener ninguna situación de conflicto de intereses de carácter financiero, académico, político o de otra índole en lo que respecta al desarrollo y publicación de esta pesquisa.

\section{REFERENCIAS BIBLIOGRÁFICAS}

1. UNICEF. Lactancia materna: consecuencia sobre la supervivencia infantil y situación mundial. [Internet] [Acceso el 08 de agosto del 2016]. Disponible en: http://www.unicef.org/spanish/nutrition/index 24824.html

2. WHO, UNICEF, IBFAN. Marketing of breast-milk substitutes: National implementation of international code. Status reports 2016. [Internet]. Geneva: Wold Health Organization; 2016. [Acceso el 08 de Agosto del 2016]. Disponible en: http://apps.who.int/iris/bitstream/10665/206008/1/9789241565325 eng.pdf?ua=1\&ua=1

3. Comité de Lactancia Materna, AEP. Lactancia materna en niños mayores o "prolongada". [Internet]. 2015 [Acceso el 08 de agosto del 2016]. Disponible en: http://www.aeped.es/comite-lactancia-materna/documentos/lactancia-maternaen-ninos-mayores-o-prolongada 
4. Vargas R. Lactancia materna y desarrollo del lenguaje. En: Terapeuta del lenguaje [Internet]. San José, C.R.: Vargas; c2013-2016. [actualizado Jun 2014, consultado 16 mayo 2016]. Disponible en:

http://www.terapeutadellenguaje.com/lactancia-materna-y-desarrollo-del-lenguaje/\#respond

5. WHO. Alimentación del lactante y del niño pequeño. [Internet]. [Acceso el 08 de agosto del 2016]. Disponible en: http://www.who.int/mediacentre/factsheets/fs342/es/

6. Mosby's Medical Dictionary. 9th edition. [Internet]. Elservier. 2009. Profesional de la salud. [Acceso el 09 de Agosto del 2016]. Disponible en: http://medical-dictionary.thefreedictionary.com/health+professional

7. Labbok, M.H. y Starling, A. Definitions of Breastfeeding: Call for the Development and Use of Consistent Definitions in Research and Peer-Reviewed Literature. 2012. Breastfeed Med [Internet]. 2012 [Acceso el 08 de julio del 2016]; 7

(6). Disponible en: http://online.liebertpub.com/doi/abs/10.1089/bfm.2012.9975\#/doi/abs/10.1089/bfm.2012.9975

8. Cabello, J.B. por CASPe. Plantilla para ayudarte a entender un Ensayo Clínico. CASPe. Guías CASPe de Lectura Crítica de la Literatura Médica. [Internet]. Alicante: CASPe; 2005. Cuaderno I. p.5-8. [Acceso el 09 de julio del 2016]. Disponible en:

http://www.redcaspe.org/system/tdf/materiales/plantilla ensayo_clinico_v1_0.pdf?file=1\&type=node\&id=158\&force=

9. Cabello, J.B. por CASPe. Plantilla para ayudarte a entender una Revisión Sistemática. CASPe. Guías CASPe de Lectura Crítica de la Literatura Médica. [Internet]. Alicante: CASPe; 2005. Cuaderno I. p.13-17. [Acceso el 09 de julio del 2016]. Disponible en:

http://www.redcaspe.org/system/tdf/materiales/plantilla_revision.pdf?file=1\&type=node\&id=154\&force=

10. Cabello, J.B. por CASPe. Plantilla para ayudarte a entender Estudios de Cohortes. CASPe. Guías CASPe de Lectura Crítica de la Literatura Médica. [Internet]. Alicante: CASPe; 2005. Cuaderno II. p.23-27. [Acceso el 09 de julio del 2016]. Disponible en: http://www.redcaspe.org/system/tdf/materiales/cohortes11.pdf?file=1\&type=node\&id=157\& force $=$

11. Cabello, J.B. por CASPe. Plantilla para ayudarte a entender un Estudio de Casos y Controles. CASPe. Guías CASPe de Lectura Crítica de la Literatura Médica. [Internet]. Alicante: CASPe; 2005. Cuaderno II. p.13-19. [Acceso el 09 de julio del 2016]. Disponible en:

http://www.redcaspe.org/system/tdf/materiales/casos_y controles.pdf?file=1\&type=node\&id=156\&force $=$

12. Rojas-Valenciano L, Cubero-Alpízar C y Leiva-Díaz V. Investigación secundaria: Utilización de la mejor evidencia en la práctica clínica. San José: CIEBE, Universidad de Costa Rica; p. 166-167.

13. Fernández-Manrique MG. Efecto de la lactancia materna en el desarrollo de niños y niñas de 18 a 4 años.[Tesis] [Quito-Ecuador]: Universidad San Francisco de Quito; 2014. 30 p. Disponible en http://repositorio.usfq.edu.ec/bitstream/23000/3655/1/111219.pdf

14. Tintaya-Peña, V. Barreras de lactancia materna y asociación de sus prácticas con el desarrollo psicomotor en niños de 6 a 24 meses, Cerro Papa - Villa el Salvador. [Tesis] [Peru]: Universidad Nacional Mayor de San Marcos; 2015. Disponible en http://cybertesis.unmsm.edu.pe/handle/cybertesis/4393 
15. Demestre X, Schonhaut L, Morillas J, Martínez-Nadal S, Vila C, Raspall F, et al. Riesgo de déficit en el desarrollo en los prematuros tardíos: evaluación a los 48 meses mediante el Ages \& Stages Questionnaires. An Pediatr (Barc) [Internet]. 2016. [Acceso el 08 de julio del 2016]; 84 (1):39-45. DOI: 10.1016/j.anpedi.2015.02.017. Disponible en http://www.analesdepediatria.org/es/riesgo-deficits-el-desarrollo-los/articulo/S1695403315000922/

16. Oddy W, Li J, Robinson M, Whitehouse A. Breastfeeding and early child development: a prospective cohort study. Acta Paediatr.[Internet]. 2011 Jul [Acceso el 08 de julio del 2016]; 100 (7): 992-9. Citado en PubMed; PMID: 21299610. DOI: 10.1111/j.1651-2227.2011.02199.x. Disponible en: https://www.ncbi.nlm.nih.gov/pubmed/21299610

17. Belfort MB, Rifas-Shiman SL, Kleinman KP, Guthrie LB, Bellinger DC, Taveras EM, et al. Infant Feeding and Childhood Cognition at Ages 3 and 7 Years. Effects of Breastfeeding Duration and Exclusivity. JAMA Pediatr. [Internet]. 2013 Set. [Acceso el 09 de julio del 2016]; 167(9):836-44. Citado en PubMed; PMID: 23896931. DOI: 10.1001/jamapediatrics.2013.455. Disponible en: https://www.ncbi.nlm.nih.gov/pubmed/23896931

18. Peters KE, Huang J, Vaughn MG, Witko C. Does breastfeeding contribute to the racial gap in reading and math test scores? Ann Epidemiol. [Internet] 2013 Oct. [Acceso el 09 de julio del 2016]. Citado en PubMed; PMID: 23880156. DOI: 10.1016/j.annepidem.2013.06.091. Disponible en: https://www.ncbi.nlm.nih.gov/pubmed/23880156

19. Ou X, Andres A, Cleves MA, Pivik RT, Snow JH, Ding Z. et al. Sex-specific association between infant diet and white matter integrity in 8-y-old children. Pediatr Res [Internet]. 2014 Dic. [Acceso el 09 de julio del 2016]. Citado en PubMed; PMID: 25167204. DOI: 10.1038/pr.2014.129. Disponible en:

https://www.ncbi.nlm.nih.gov/pubmed/25167204

20. Smith JM. Breastfeeding and language outcomes: a review of literature. Journal of Communication Disorders [Internet]. 2015. [Acceso el 14 de junio del 2016]; 57:29-40. DOI: 10.1016/j.jcomdis.2015.04.002. Disponible en: http://www.sciencedirect.com/science/article/pii/S0021992415000258

21. Delgado C, MatijasevichI A. Breastfeeding up to two years of age or beyond and its influence on child growth and development: a systematic review. Cad Saude Publica [Internet]. 2013 Feb. [Acceso el 08 de julio del 2016]; 29(2): 24356. Citado en PubMed; PMID: 23459811. Disponible en: https://www.ncbi.nlm.nih.gov/pubmed/23459811

22. Walfisch A, Sermer C, Cressman A, Koren G. Breast milk and cognitive development - the role of confounders: a systematic review. Obstetrics and gynaecology [Internet]. 2013 Ago. [Acceso el 09 de julio del 2016]; 3(8)DOI: 10.1136/bmjopen-2013-003259. Disponible en http://bmjopen.bmj.com/content/3/8/e003259.full.pdf

23. Espinosa-Cevallos YB, Maldonado-Pacheco JE. Lactancia materna y su asociación con el rendimiento académico en estudiantes de $6^{\circ}$ y $7^{\circ}$ años de educación básica en la Unidad Educativa San José la Salle de la ciudad de Latacunga, Cotopaxi - Ecuador en el primer quimestre del periodo escolar 2015 -2016. [Tesis]. [Cotopaxi-Ecuador]: Pontificia Universidad Católica del Ecuador; 2016. 150 p. Disponible en: http://repositorio.puce.edu.ec/handle/22000/10474

24. Rondón R, Zambrano G, Guerra ME. Relación de la lactancia materna y el desarrollo Dento-Buco-Máxilo-Facial: Revisión de la literatura latinoamericana. Revista Latinoamericana de Ortodoncia y Odontopediatría [Internet]. 2012. [Acceso el 08 de julio del 2016]; 1-27. Disponible en: http://ortodoncia.ws/publicaciones/2012/pdf/art20.pdf 
25. Cigüeñas-Raya EM. Influencia de la lactancia materna en la prevención de maloclusiones, en infantes de 0 a 36 meses de edad. [Tesis] [Perú]; Universidad Nacional Mayor de San Carlos; 2013. Disponible en:

http://200.62.146.130/bitstream/cybertesis/3080/3/Cigue $\%$ C3\%B1as re.pdf

26. Montoya V, Córdova M, del Cisne L. Riesgo de sustitución de la lactancia natural por leche artificial y su relación con el estado de salud del lactante que acude al servicio de pediatría periodo abril 2012 a septiembre del 2012. [Tesis] [LojaEcuador]; Universidad Nacional de la Loja; 2012. 103 p. Disponible en:

http://dspace.unl.edu.ec/jspui/handle/123456789/6673

27. Hörnell, A, Lagström, H, Lande, B y Thorsdottir, I. Breastfeeding, introduction of other foods and effects on health: a systematic literature review for the 5th Nordic Nutrition Recommendations. Food Nutr Res. [Internet]. 2013 Abr. [Acceso el 08 de julio del 2016]; 12:57. Citado en PubMed; PMID: 23589711. DOI: 10.3402/fnr.v57i0.20823. Disponible en: https://www.ncbi.nlm.nih.gov/pubmed/23589711

28. Whitehouse AJ, Robinson M, Li J., Oddy WH. Duration of breast-feeding and language ability in middle childhood. Paediatr Perinat Epidemiol. 2011 Jan. [Acceso el 09 de julio del 2016]; 25(1):44-52. Citado en PubMed; PMID: 21133968. DOI: 10.1111/j.1365-3016.2010.01161.x. Disponible en: http://www.ncbi.nlm.nih.gov/pubmed/21133968

29. Quigley MA, Hockley C, Carson C, Kelly Y, Renfrew MJ, Sacker A. Breastfeeding is Associated with Improved Child Cognitive Development: A Population-Based Cohort Study. J Pediatr [Internet]. 2012 Ene. [Acceso el 08 de julio del 2016]; 160(1); 25-32. Citado en PubMed; PMID: 21839469. DOI: 10.1016/j.jpeds.2011.06.035. Disponible en https://www.ncbi.nlm.nih.gov/pubmed/21839469

30. Werker JF, Hensk TK. Critical periods in speech perception: New directions. Annu Rev Psychol.[Internet]. 2015 Ene [Acceso el 14 de junio del 2016]; 3(66):173-96. Citado en PubMed; PMID: 25251488. DOI: 10.1146/annurev-psych010814-015104. Disponible en: https://www.ncbi.nlm.nih.gov/pubmed/25251488

31. Cockerham-Colas L, Geer L, Benker K, Joseph MA. Exploring and influencing the knowledge and attitudes of health professionals towards extended breastfeeding. Breastfeed Med [Internet]. 2012 Jun [Acceso el 10 de agosto del 2016]; 7 (3): 143-50. Citado en PubMed; PMID:21854294. DOI: 10.1089/bfm.2011.0027 Disponible en: https://www.ncbi.nlm.nih.gov/pubmed/21854294

32. Rodríguez-García R. Aproximación antropológica a la lactancia materna. Antropología Experimental [Internet]. 2015 [Acceso el 10 de agosto del 2016]; 15: 407-429. DOI: 10.17561/rae.v0i15.2620. Disponible en: http://revistaselectronicas.ujaen.es/index.php/rae/article/view/2620

33. Dettwyler K. A time to wean. Breastfeeding Abstracts [Internet]. 1994 Ago [Acceso el 10 de agosto del 2016]; 14 (1): 3-4. Disponible en: http://www.naturalchild.org/guest/katherine dettwyler.html

34. Tedder, J. The Roadmap to Breastfeeding Success: Teaching Child Development to Extend Breastfeeding Duration. J Perinat Educ [Internet]. 2015 [Acceso el 14 de junio del 2016]; 24 (4):239-248. Citado en PubMed; PMID: PMC4718010. DOI: 10.1891/1058-1243.24.4.239. Disponible en: http://www.ingentaconnect.com/content/springer/jpe/2015/00000024/00000004/art00006 